\title{
Infectious diarrhea with sepsis symptoms from Yokenella Regensburgei
}

\begin{abstract}
Yokenella regensburgei of the family Enterobacteriaceae is a rare clinical isolate. Only six infection cases in humans have been reported previous to the present. We report a case of infectious diarrhea with sepsis symptoms in a 17 year old patient with $Y$. regensburgei.

\section{Background}

Yokenella regensburgei is an opportunistic human pathogen, a gram-negative, oxidase negative, motile rod, fermentative bacterium. ${ }^{1}$ whose infections are rarely reported in humans. Only 6 cases have been previously reported regarding septic knee ${ }^{2}$, urinary tract infection ${ }^{3}$, transient bacteraemia ${ }^{2}$, perimalleolar ulcer $^{4}$, cellulitis ${ }^{5}$, septic shock ${ }^{6}$ and enteric fever ${ }^{7}$ similar to the present case.

It belongs to the family Enterobacteriaceae ${ }^{8}$ and it is biochemically similar to the bacterium Hafnia alvei with their difference being that $H$. alvei is resistant to colistin and Voges-Proskauer Test (VP) negative, whereas Y. regensburgei has the opposite characteristics $^{8,9}$. The National Institutes of Health in Japan identified Y. regensburgei as NIH biogroup 9 and the Centers for Disease Control and Prevention in Atlanta as enteric group 45, proposing the name Koserella trabulsii. The name Yokenella regensburgei proposed by Kosako et al. ${ }^{1}$ finally prevailed over $K$. trabulsii. ${ }^{1,2}$. The bacterium has been isolated from intestinal tracks of insects and reptiles, well water and salad. ${ }^{7}$ as well as from the following human anatomical sites: $\operatorname{blood}^{6}$, faecal samples ${ }^{8}$, upper respiratory tract, urine..$^{2,3}$ and knee fluid. ${ }^{9,10}$ The small number of the cases reported with Y.regensburgei as a pathogen is responsible for the shortage of epidemiological and clinical information. Here we present a case from Y.regensburgei which is to our current knowledge the first case reported in Greece.
\end{abstract}

Volume 5 Issue I - 2017

\author{
Milori A,' Miliori E, ${ }^{2}$ Gkatsis ${ }^{1,3}$ Koletti GB, ${ }^{3}$ \\ Vogiatzi A, ${ }^{3}$ Paulou $A^{\prime}$ \\ 'Department of Microbiology, General Hospital Messolonghi, \\ Greece \\ ${ }^{2} S c h o o l$ of Medicine National and Kapodistrian University of \\ Athens, Greece \\ ${ }^{3}$ Department of Internal Medicine, General Hospital \\ Messolonghi, Greece
}

Correspondence: Milori A, Department of Microbiology, General Hospital Messolonghi, rysostomoy Smyrnis 2, Melissia, Greece, Tel 6972199684, Email arianamilori@gmail.com

Received: April 20, 2017 | Published: May 24, 2017

\section{Case report}

A 17-year old male athlete was transported to General Hospital of Messolonghi from the community Health Centre of Astakos with 6-7 daily episodes of diarrhea, fever up to $39.8 \mathrm{oC}$ and chills. The patient reported that these smptoms emerged three days before his arrival to the Health Centre of Astakos, three hours after physical exercise and that the fever would not subside with the consumption of paracetamol and mefenamic acid. He arrived to General Hospital of Messolonghi on the fourth day of the symptoms. Physical examination with deep palpation of abdomen showed sensitivity in the entire abdominal wall and increased bowel sounds. A systemic examination revealed nothing of note. Patient's medical history showed only cefuroxime allergy. His vital signs were: blood pressure $135 / 60 \mathrm{~mm} / \mathrm{Hg}$, pulse rate $105 / \mathrm{min}$, Oxygen Saturation (SO2) $98 \%$ and body temperature $39.0^{\circ} \mathrm{C}$. Upper and lower abdominal sonography results were normal. Laboratory examination showed leukocytosis (WBC 13,270/mml-3), neutrophilia (76.7\%, normal range 40-74\%), absolute neutrophil count 10,180/mml-3, lymphocytes $11.8 \%$ (19-48\%), monocytes 7\% (3.4-9\%), eosinophils $1.5 \%(0-7 \%)$, increased C- Reactive Protein (CRP) $18.76 \mathrm{mg} / \mathrm{dl}(0-$ $0.8 \mathrm{mg} / \mathrm{dl}$ ), hemoglobin $14,2 \mathrm{mg} \mathrm{dl}-1$ and mean corpuscular volume (MCV) 78,5fL. The rest of the biochemical test results (glucose, urea, creatinine, uric acid, bilirubin, serum glutamic oxaloacetic transaminase (SGOT), serum glutamic pyruvic transainase (SGPT), alkaline phosphatase, gamma-glutamyl transferase (G-GT), amylase, albumin, total serum protein, creatine phosphokinase (CPK), lactate dehydrogenase (LDH), Calcium (Ca), Sodium (Na), Potassium (K) , total cholesterol (TC), high-density lipoprotein, low-density lipoprotein, Iron (Fe), immunoglobulin igG, igM and $\operatorname{IgA}$ ) were normal. The widal test for typhoid and wright tests were negative. Blood smear examination showed neutrophils with toxic granulation, and white blood cell count differential showed band neutrophils $12 \%$, neutrophils $67 \%$, lymphocytes $12 \%$, monocytes $7 \%$, eosinophils $2 \%$. Urine culture showed no growth. General stool examination showed a large amount of white blood cells, erythrocytes and mucous. Parasitological examinations" results for gastro-intestinal protozoa, nematodes roundworms and flatworms were negative. X-ray of the chest was normal.

Two stool culture samples were prepared in two days and after a 48-hour incubation, bacterial growth was observed on SS agar for both samples. This bacterium was non-lactose fermenting with opaque colony morphology unlike the transparent colonies of shigella. Biochemical testing of the microorganism showed that the bacterium was weakly positive for catalase and negative for oxidase, urease, indole and $\mathrm{H} 2 \mathrm{~S}$. The identification testings were performed by VITEK 2 Compact automated system (Bio Merieux, France) and identified the bacterium as Yokenella regensburgei. Biochemical tests of Vitek - 2 are presented in Table 1. The stool cultures were negative for the following enteropathogens: Salmonella, Shigella, Campylobacter, Enterohemorrhagic, Escherichia coli and Yersinia enterocolitica.

Antimicrobial susceptibility test was performed using Disk diffusion: Mueller-Hinton agar (MHA) method according to the Clinical and Laboratory Standards Institute (CLSI) guidelines (Zone Diameter and Minimal Inhibitory Concentration (MIC) Interpretive Standards for Enterobacteriaceae). ${ }^{11}$. The microorganism was resistant 
to ampicillin and colistin, intermediate to amoxicillin/clavulanic acid and sensitive to cefotaxime, amikacin, gentamicin, cefuroxime, cefixime, ciprofloxacin, imipenem, meropnem, aztreonam and trimethoprim/sulfamethoxazole.

The patient received treatment with cefixime and his symptoms were resolved. After 3 days of hospitalization he was dismissed, continued the treatment with cefixime for six more days and was recommended for a follow-up 10 days after the end of the treatment. Clinical examination showed no evidence of relapse.

\section{Conclusion}

The track of the pathogen transmission remains unclear., ${ }^{6,7}$ The patient of this case reported that three hours before the symptoms (abdominal pain and diarrhea) emerged, he had returned home after his sport activity, boiled an egg and consumed it. There appears no correlation between this incident and the other cases reported ${ }^{2-7}$. Although in five of the six previous cases the patients were immune suppressed (alcohol consumption, adenocarcinoma, chronic renal failure, diabetes mellitus and use of steroids were reported $)^{2-6}$, the patient of the present case had no such medical history and seemed to be immunocompetent as he is an young athlete, there was no primary immune deficiency referred, he receives no immunosuppressive drugs and no chronic illness or recurrent infections were referred. The previous cases involved patients of an older age. ${ }^{2-6}$ Only one case has been reported of a younger person (aged 5) who was immunocompetent previous to the present patient. ${ }^{7}$

The patient met the SIRS (Systemic Inflammatory Response Syndrome) criteria which involve two or more of the following: body temperature over $38 \mathrm{oC}$ or under $36 \mathrm{oC}$, pulse rate over $90 / \mathrm{min}$, respiratory rate over 20 breaths $/ \mathrm{min}$ or $\mathrm{PaCO} 2$ under $32 \mathrm{mmHg}$, WBC over 12000 or less than $4000 \mathrm{mml}-3 .{ }^{12}$ The patient showed body temperature $39.8 \mathrm{oC}$, pulse rate $105 / \mathrm{min}$ and $\mathrm{WBC} 13270 \mathrm{mml}-3$.

Yokenella regensburgei is probably a potentially dangerous pathogen that has been rarely isolated from humans. ${ }^{7}$ It is highly possible that some cases of Y.regensburgei have not been identified, as the stool culture results showed opaque colony morphology on SS agar, which does not indicate a common enteropathogen colony, and therefore we do not continue to the identification process.

\section{Acknowledgements}

None.

\section{Conflicts of interest}

There is no conflict of interest.

\section{Funding}

None.

\section{References}

1. Kosako Y, Sakazaki R, Yoshizaki. Yokenella regensburgei gen. nov., sp. nov.: a new genus and species in the family Enterobacteriaceae. Jpn J Med Sci Biol. 1984;37(3):117-124.

2. Abbott SL, Janda JM. Isolation of Yokenella regensburgei ("Koserella trabulsii") from a patient with transient bacteremia and from a patient with a septic knee. J Clin Microbiol. 1994;32(11):2854-2855.

3. Zahid S Aziz. Emerging of Yokenella regensburgei as Uropathogen: First Report. Journal of Biology Agriculture and Healthcare. 2015;5(7):74 76.

4. Fajardo Olivares M, Blanco Palenciano J, et al. Yokenella regensburgei infection in a perimalleolar ulcer. Med Clin (Barc). 2005;125(9):358-359.

5. Bhowmick T, Weinstein MP. A Deceptive Case of Cellulitis Caused by a Gram-Negative Pathogen. J Clin Microbiol. 2013;51(4):1320-1323.

6. M Fill, J Stephens. Abdominal Abscess and Septic Shock Secondary to Yokenella regensburgei. J Infect Dis. 2009;9(1):1.

7. Jain S, Gaind R, Gupta KB, et al. Yokenella Regensburgei infection in India mimicking enteric fever. J Med Microbiol. 2013;62(6):935-939.

8. Stock I, Sherwood KJ, Wiedemann B. Antimicrobial susceptibility patterns, [beta]-lactamases, and biochemical identification of Yokenella regensburgei strains. Diagn Microbiol Infect Dis. 2004;48(1):5-15.

9. Kosako Y, Sakazaki R, Huntley Carter GP, et al. Yokenella regensburgei and Koserella trabulsii are subjective synonyms. Int J Syst Bacteriol. 1987;37(2):127-129.

10. Hickman Brenner FW, Huntley Carter GP, Fanning GR, et al. Koserella trabulsii, a new genus and species of Enterobacteriaceae formerly known as Enteric Group 45. J Clin Microbiol. 1985;21(1):39-42.

11. CLSI. Performance Standards for Antimicrobial Susceptibility Testing; 22nd Informational Supplement M100-S22. CLSI. 2014;32(3):1-188.

12. Singer M, Deutschman CS, Seymour CW, et al. The Third International Consensus Definitions for Sepsis and Septic Shock (Sepsis-3). JAMA. 2016;315(8):801-810. 